\title{
A NEURAL NETWORK SYSTEM WITH REINFORCEMENT LEARNING TO CONTROL A DYNAMIC ARM MODEL
}

\author{
Ilkay Ulusoy, Mohamad PARnianPOUR*, NeCiP BERME**, SHEldON R. SimON***
}
Biomedical Engineering Center, `Department of Industrial, Welding and Systems Engineering, **Department of Mechanical Engineering, ${ }^{\star \star}$ Department of Orthopaedic Surgery, The Ohio State University, Ohio, USA

\begin{abstract}
A neural network system is presented for controlling a two-link dynamic arm model where the task is to move the arm from any initial position to any final position in the sagittal plane. The controller produces joint torque-time profiles that begin and end with equilibrium values at the initial and final positions, respectively. A memory type neural network is trained by supervised learning methods to predict the joint's static equilibrium torque values corresponding to joint angles. A reinforcement learning network is used to determine the parameters needed for synthesis of the torquetime profiles for each joint. The reinforcement signal is computed based on the distance between the desired end point position and velocity and the states achieved based on the generated torque profiles. The general pattern of the torque-time plots is decided a priori according to the literature. The methods of training and an illustrative example of the algorithm's performance are presented.
\end{abstract}

Biomed Eng Appl Basis Comm, 2001 (June); 13: 117-123.

\section{INTRODUCTION}

Movement control mechanism in human body deals with highly nonlinear biological systems. The nonlinearity is due to the intrinsic second-order differential equations that result from formulation of equations of motion in addition to the nonlinearity due to the endowed sensory and motor systems controlling the skeletal system. There are attempts to model the biological controllers by using engineering methods. Some very common engineering methods such as feed-

Received: Feb. 25, 2001; accepted: April 10, 2001

Correspondence: Mohamad Parnianpour, Ph.D.

Department of Industrial, Welding and Systems Engineering, The Ohio State University, 1971 Neil Ave, Columbus, Ohio 43210, USA

E-mail: Parnianpour.1@osu.edu back and feed-forward control can also be found in the literature. In order to control biological systems, an accurate controller that can adapt to the changing environment is required. Therefore, adaptive control is quite suitable for such control problems. However, according to Gullapalli [3] since nonlinear adaptive control builds upon principles of linear control, adaptive algorithms developed by traditional engineering methods may not be appropriate for understanding of control of biological systems.

In recent years, learning to control is thought to be more efficient than adaptive control methods since learning mechanisms may better approximate the actual physiology when external rules introduced to the learning system emerge from the context of observed biological system behavior. In the general definition of learning to control a task, the learning system plays the role of a controller whose actions serve as control inputs to a process. The controller generates control actions based on its inputs, which may include the task 
specification and possible feedback. The output of the process is estimated or measured by a set of intrinsic or extrinsic sensors. Finally, guided by some form of training information, the controller learns to generate appropriate control actions for performing the specified task.

For such a heuristic solution, artificial neural networks (ANNs) are very effective because of their computational power. Neural networks can approximate various functions that are relevant to control purposes and can be implemented using the supervised and/or reinforcement learning methods. In supervised learning, the controller should be trained off-line using the input-output example pairs of the task to be learned. In reinforcement learning algorithms, the learning system explores the correct actions by itself and a reinforcement signal sent from the environment is used to correct the learning system's output. Reinforcement learning is especially used for direct (forward dynamics) optimal control problems [8].

This study presents a neural network system (Figure 1) with one reinforcement learning network (NN1) and one supervised learning network (NN2) to control a two link dynamic arm model where the task is to move the arm from an initial position to a final position by synthesizing the appropriate torque profiles to drive the system

\section{METHODS}

The two-link arm's dynamic equations of motion are simulated based on the anthropometric data [10] presented in Table 1. The passive and active viscoelasticity of the system have been considered to achieve a stable simulation. For the purpose of simplicity, the actual muscle models are not included in the arm model. Instead, the net muscular joint torque values are considered as controlling signals (Figures 1-3). The planar movement is achieved by varying the joint torque values from the equilibrium values at the initial and final positions according to the patterns of torque-time profiles produced by the network while integrating the equations of motion. The general pattern of the torquetime is decided according to the available data in the literature and the parameters required to represent the actual torque profiles for each movement are found by the neural network system after the necessary amount of training.
The two-link arm (Figure 2) is modeled by its dynamic equations of motion (Equation 1) where the movement is performed in the sagittal plane within the physiological joint angle $\left(-90^{\circ}<\theta_{1}<90^{\circ}\right.$ and $\left.0^{\circ}<\theta_{2}<180^{\circ}\right), \dot{\theta}$ and $\ddot{\theta}$ being angular velocity and acceleration vectors respectively. $\tau$ is the joint torque vector, $[\mathrm{H}(\theta)]$ is the inertia matrix, $G(\theta)$ is the gravity term and $C(\theta, \dot{\theta})$ is the coriolis and centripetal term [5]. Here, knowing the joint moments, the equations of motion are integrated for link angular velocity and displacement.

$$
[H(\theta)] \ddot{\theta}=\tau-G(\theta)-C(\theta, \dot{\theta})
$$

The controller system is modeled by two neural networks (Figure 1). A multi-layer perceptron (Figure 4) with one hidden layer of 50 units and one output layer of two units, all are nonlinear units (Figure 5), is built to store the arm static equilibrium torque values corresponding to the arm joint angles and is trained by the back-propagation algorithm [4] before it is actually used in the system. Inputs to this look-up table type of memory network are the joint angles and the outputs are the static equilibrium joint torque values. The second network is also built as a multi-layer network (Figure 6) with two hidden layers of 50 nonlinear units in each and one output layer of 6 Stochastic Real Valued (SRV) units (Figure 7), and is trained by the SRV reinforcement learning algorithm [2]. The inputs to this network are the sines and cosines of both the initial and desired final arm joint angles and the outputs are six parameters $\left(p_{i}, m_{i}\right.$, and $t_{i}$ for $i^{\text {th }}$ joint $)$ which will be used to construct a torque-time profile for each joint using the torque-time schema decided a priori (Figure 3 ) according to the experimental results showing that during reaching, humans produce bell-shaped speed profile [1]. The torque-time schema is generated by the summation of a linear function and a sinusoidal function. The equilibrium torque needed at the initial and final arm positions defines the former, and the latter is determined by three parameters: ( $t_{i}$ : the duration of positive torque, $\mathrm{p}_{\mathrm{i}}$ : the peak positive torque, $\mathrm{m}_{\mathrm{i}}$ : peak negative torque).

After training, for a desired movement, i.e. the desired initial and final positions introduced to the networks as inputs, the initial and final position's equilibrium torque values obtained from the memory network and the parameters produced by the controller network are used to construct the specific torque-time

Table 1. The anthropometric parameters of the two-link arm based on [10].

\begin{tabular}{lllll}
\hline Link & $\begin{array}{l}\text { Mass } \\
(\mathrm{kg})\end{array}$ & $\begin{array}{l}\text { Length } \\
(\mathrm{m})\end{array}$ & $\begin{array}{l}\text { Center of mass } \\
(\mathrm{m})\end{array}$ & $\begin{array}{l}\text { Moment of inertia } \\
\left(\mathrm{kg}-\mathrm{m}^{2}\right)\end{array}$ \\
\hline 1 & 1.96 & 0.3348 & 0.14 & 0.02 \\
2 & 1.54 & 0.4572 & 0.18 & 0.026 \\
\hline
\end{tabular}




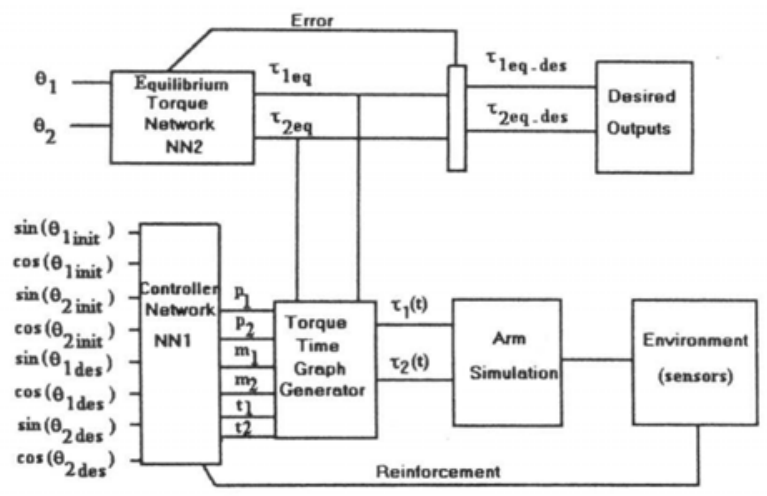

Figure 1. The controller system consisting of two neural networks for generation of torque-time patterns to achieve the movement of a two-link dynamic arm.

profiles for the given movement. Eventually, the applied torque is adjusted by NN1 to achieve synthesizing the torque profiles that move the arm from the initial position to the desired final position in the specified duration.

\section{Training}

In the memory network, which is a multi layer perceptron (Figure 4), $\xi_{k}$ is the input ' $k$ ', $V_{j}^{m}$ is a hidden unit ' $j$ ' in layer ' $m$ ' where ' $M$ ' is the output layer, $w_{\ln }$ is a connection weight from unit ' $n$ ' to unit ' $l$ '. The output of a nonlinear unit is calculated as a function of the weighted sum of inputs (Equation 2) where ' $\mathrm{f}$ ' is called as activation function (Equation 3) and ' $\mathrm{g}$ ' is the gain.

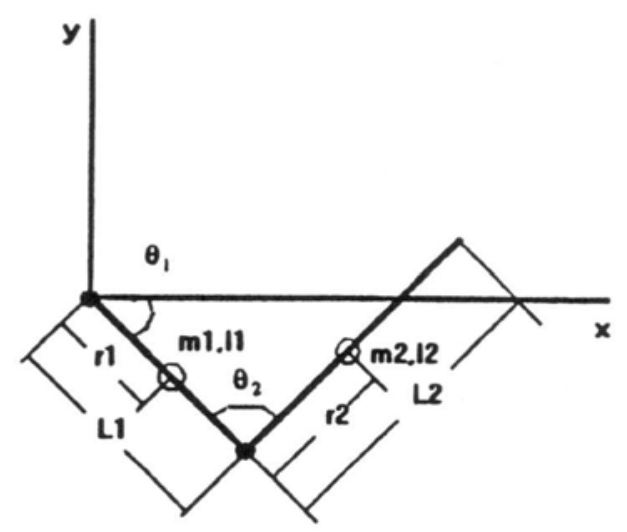

Figure 2. The arm model with the appropriate anthropometric data [10] presented in Table

$$
\begin{aligned}
& V_{j}^{m}=f\left(h_{j}{ }^{m}\right) \\
& \text { where.... } h_{j}{ }^{m}=\sum w_{j l} V_{l}^{m-1}+\theta_{t h r} \\
& f(x)=1 /(1+\exp (-g \cdot x))
\end{aligned}
$$

After finding the output for each unit in each layer starting from the input layer towards the output layer, which is usually called as a forward pass, the network outputs are compared with the desired values and errors are backpropagated. In back-propagation algorithm, the purpose is to reduce the mean square error (Equation 4), where $\zeta_{i}$ is the desired output for output unit ' $i$ ' and $O_{i}$ is the network output for that unit, and weight update is performed as in Equation 5 where ' $\eta$ ' is the learning rate.
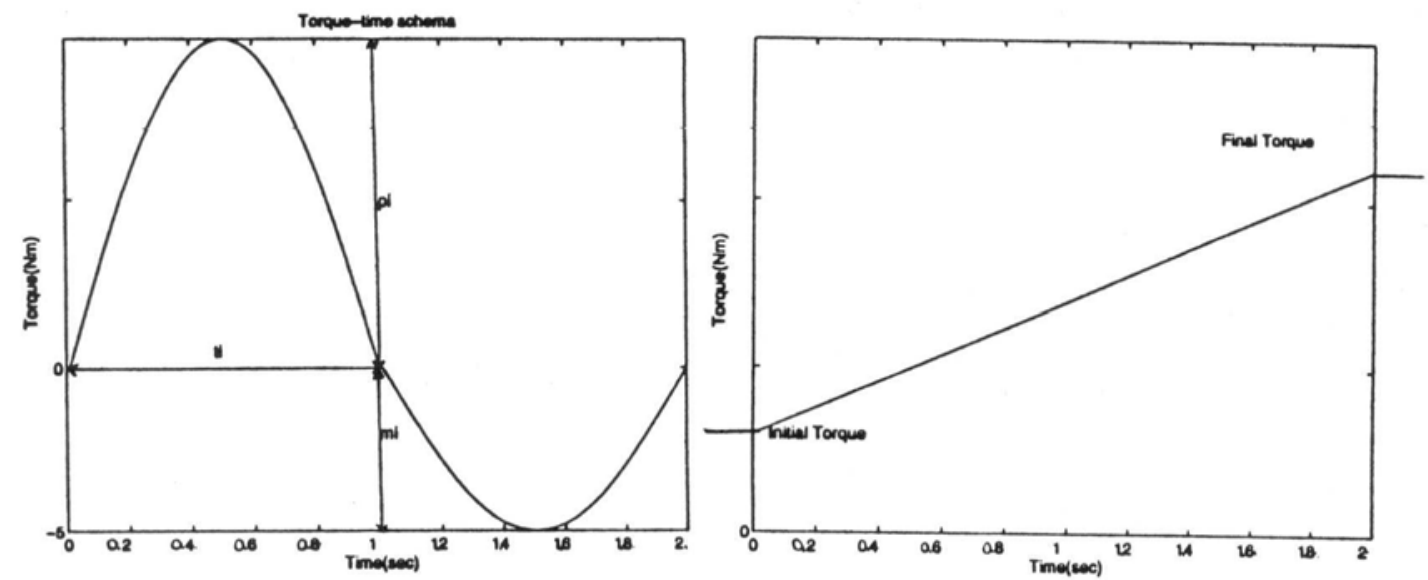

Figure 3. The torque-time schema is generated by the summation of a linear function and a sinusoidal function. The equilibrium torque needed at the initial and final arm position defines the former, while the latter is defined by three parameters: ( $t_{i}$ : the duration of positive torque, $p_{i}$ : the peak positive torque, $m_{j}$ : peak negative torque). 

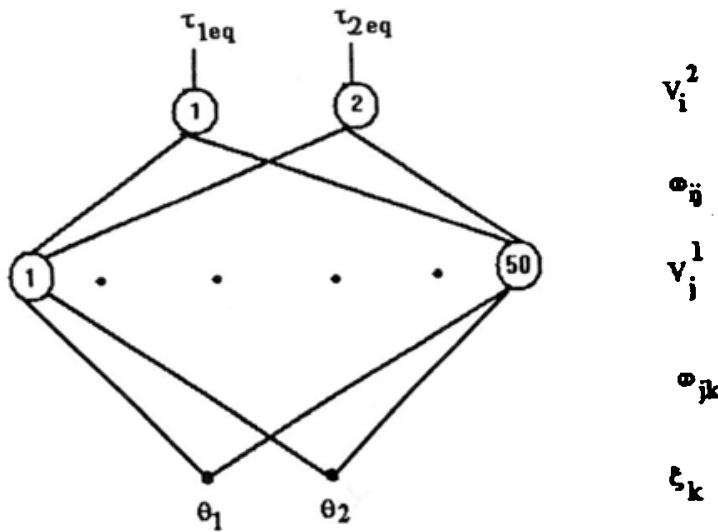

Figure 4. Memory network to learn the static equilibrium torque at any arm position.

$$
\begin{aligned}
& E(w)=1 / 2 \sum_{i}\left(\zeta_{i}-O_{i}\right)^{2} \\
& \Delta w_{\mathrm{ln}}=-\eta \partial E / \partial w_{\mathrm{ln}}
\end{aligned}
$$

In this algorithm, if unit ' $i$ ' is an output unit then weight between the output unit ' $i$ ' and the hidden unit ' $j$ ' in the previous layer is performed as in Equation 6 where $\delta_{i}=\left(\zeta_{i}-O_{i}\right) \dot{f}\left(h_{i}\right)$.

$$
\Delta w_{i j}=\eta \delta_{i} V_{j}
$$

If ' $j$ ' is a hidden unit in layer ' $m$ ' then weight between unit ' $j$ ' in hidden layer ' $m$ ' and unit ' $l$ ' in layer ' $m-1$ ' is as in Equation 7 where $\delta_{j}{ }^{m}=\dot{f}\left(h_{j}{ }^{m}\right)\left(\sum_{i} w_{i j} \delta_{i}{ }^{m+1}\right)$.

$$
\Delta w_{j l}=\eta \delta_{j}^{m} f\left(h_{l}^{m-1}\right)
$$

The memory network is trained before it is actually used in the controller system using the data obtained from the arm static equilibrium equations. During training, first the joint angle values for an arm position are introduced to the network. Then the network calculates its outputs. The outputs of the last layer are then compared with the static equilibrium torque values corresponding to the current network inputs (joint angles) and the error is propagated backwards through the network and each connection weight is updated according to the error assigned to it as a result of the back-propagation algorithm. This process is repeated until the mean square error becomes smaller than the specified tolerance limit.

The same forward pass and back-propagation algorithm are also performed for the controller network except the output layer. In the output layer, the activa-

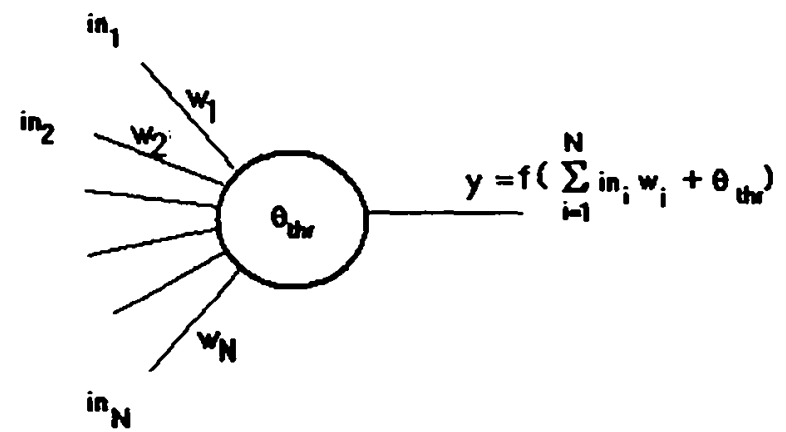

Figure 5. The input-output relationship for a nonlinear unit.

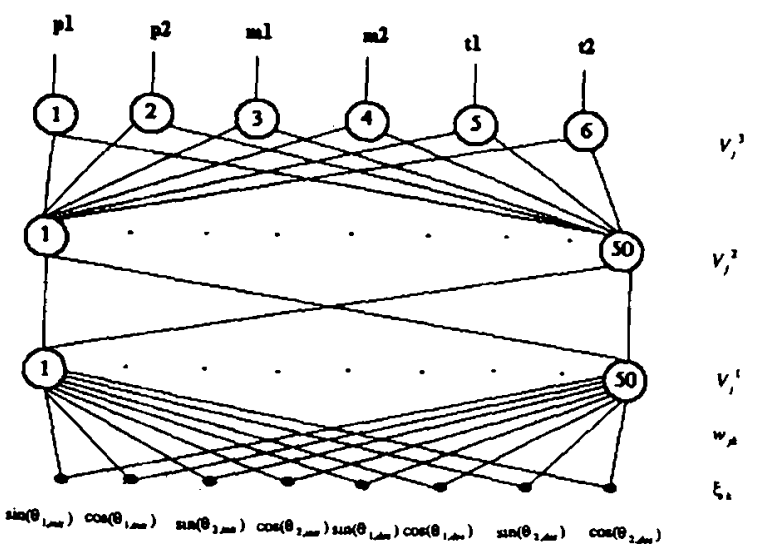

Figure 6. Controller network with two hidden layer having stochastic real valued units at its output.

tion of an SRV unit (Figure 7) will be a random selection from a Gaussian Distribution, $\psi$, specified by the mean, $\mu$, and the standard deviation, $\sigma$, calculated by the unit itself. This way network produces its own training examples randomly. And according to the reinforcement sent from the environment it either learns them or not.

The unit calculates its mean $(\mu)$ (Equation 8) and an expected reinforcement $(\hat{r})$ (Equation 9$)$ as weighted sum of its inputs and standard deviation $(\sigma)$ (Equation 10) is found as a function of $\hat{r}$ such that when $\hat{r}$ is maximum, that is when it is equal to the maximum reinforcement that could be sent from the environment (which is 1 in our case), then $\sigma$ should be zero. In this case the random search should be reduced. Otherwise, the unit should search a wide range in order to find the actions that would cause it to receive high reinforcements.

$$
\begin{aligned}
& \mu=\sum_{i=1}^{N} w_{i} \cdot i n_{i}+w_{t h r} \\
& \hat{r}=\sum_{i=1}^{N} v_{i} \cdot i n_{i}
\end{aligned}
$$




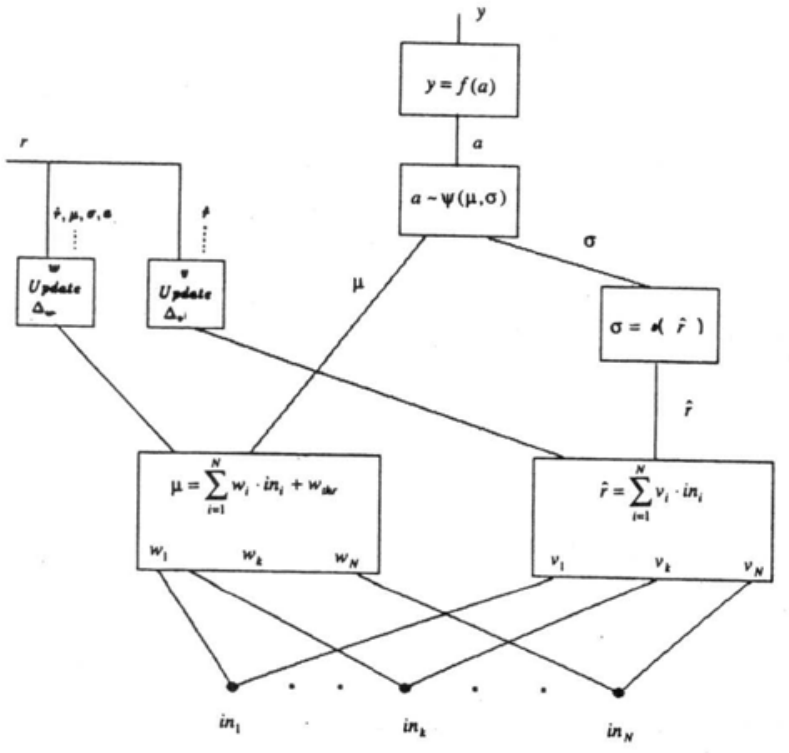

Figure 7. The stochastic real valued unit

$$
\begin{aligned}
& \sigma=s(\hat{r})=\max ((1-\hat{r}) / 5,0) \\
& a \sim \psi(\mu, \sigma) \\
& y=f(a)=1 /(1+\exp (-g . a))
\end{aligned}
$$

After the unit finds its actual output as a function of its activation (Equation 12), the response sent from the environment which was effected by the unit's output, i.e. a reinforcement signal in the $[0,1]$ range, is used by the unit to update its own weights as in Equations $13,14,15$ where $\Delta_{w}=(r-\hat{r})(a-\mu) / \sigma$ and $\Delta_{v}=r-\hat{r}$.

$$
\begin{aligned}
& \Delta w_{i}=\alpha \Delta_{w} i n_{i} \\
& \Delta w_{t h r}=\alpha \Delta_{w} \\
& \Delta v_{i}=\beta \Delta_{w} i n_{i}
\end{aligned}
$$

Thus, $w_{i}$ 's are updated such that if the unit's activation causes an environmental reinforcement, $r$, greater than the reinforcement calculated by the unit itself, $\hat{r}$, then this was a correct action and the unit's mean should be closer to this activation value. And since $\hat{r}$ is present in the unit to model $r$, the error between the two is directly used to update $v_{i}$ 's. The rest of the network is updated as in back-propagation algorithm where this time $\Delta_{w}$ 's calculated at the output units are considered as output errors, i.e. $\delta_{i}$ 's in Equation 6. At the beginning of the training, the unit has a high search activity but towards the end of learning, unit's standard deviation becomes zero and output becomes the mean.

Training of the controller network is performed on-line where joint angle sines and cosines of the ran-

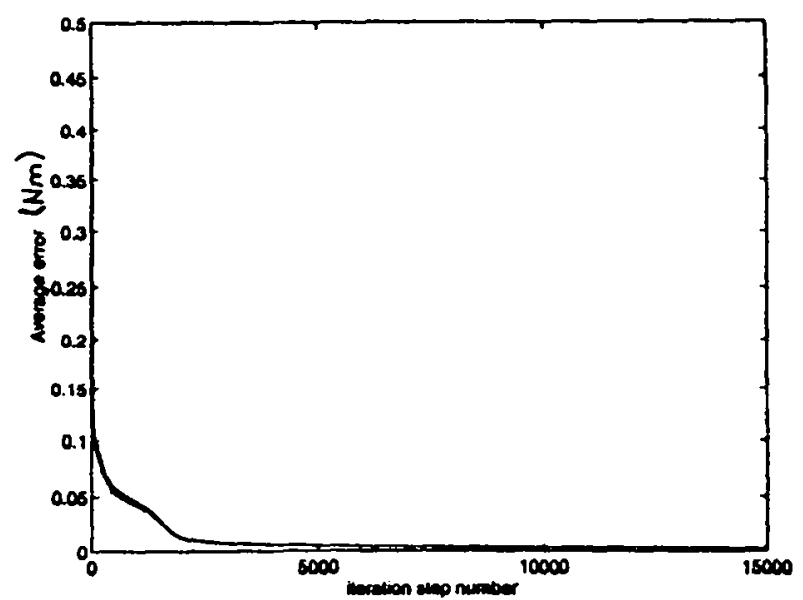

Figure 8. Average Error versus training cycle number for memory network.

domly selected initial and final positions are introduced to the network. The network randomly produces its outputs. These outputs and the equilibrium torque values obtained from the memory network, are then used to built the torque-time profiles which are then applied to the arm model. The forward dynamics equations are solved for each time interval of 0.01 seconds and the angular acceleration obtained at each time step is integrated once and twice to obtain angular velocities and joint angles for the next time step respectively. At the end of movement, the difference between the arm position and the desired final position and the endpoint tangential velocity are used to evaluate the performance as a reinforcement signal which can have continuous values in the interval [0,1] (Equation 16). After the network weights are updated, another random initial-final position pair is selected and the same procedure is followed for many times until a high reinforcement value is received from the environment for most of the movements.

$$
r=\max (1-d-v, 0)
$$

where

$$
\begin{aligned}
& d=\sqrt{\left(x_{\text {end_point }}-x_{\text {desired }}\right)^{2}+\left(y_{\text {end_point }}-y_{\text {desired }}\right)^{2}} \\
& \text { and } v=\sqrt{v_{x, \text { end_point }}{ }^{2}+v_{y, \text { end_point }}{ }^{2}}
\end{aligned}
$$

Thus, during training the network produces some random outputs by itself and evaluates the performance for appropriate reinforcement. According to the reinforcement being sent from the environment it will learn the output that corresponds with the desired outcome and abandon those patterns that do not.

\section{RESULTS}




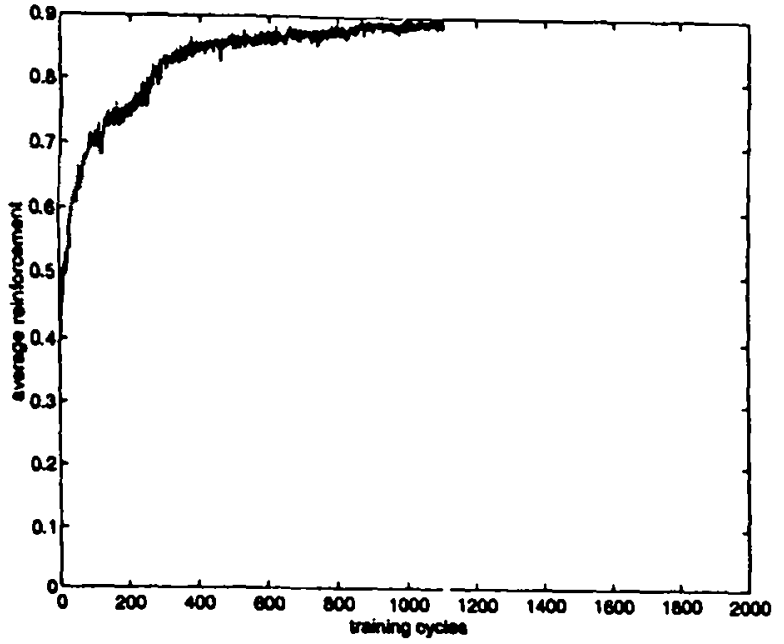

Figure 9. Reinforcement signal versus training cycle for controller network during training of a movement shown in Figure 10.

The memory network is trained for 20 different joint angle-torque pairs. After 10000 iterations, the error between the network outputs and the desired outputs averaged among training examples (20 pairs in this case) is found to be less than $0.02 \mathrm{Nm}$ (Figure 8).

The controller network is trained by repeating the cycle explained in the previous section for $1,000,000$ times. The movement duration is taken to be 2 seconds and the max torque peak is taken as $15 \mathrm{Nm}$. Towards the end of training, the exploratory behavior vanishes and the network finds a controlling rule which was able to perform the reaching movement from any initial point to any final point within the working region (for the specific 2 seconds duration of movement under study). The reinforcement signal averaged over 50 successive cycles is given in Figure 9. After training, the standard deviations are made zero and randomly selected input combinations are introduced to the network. For most of the movements, the final endpoint velocity is zero and the final distance is less than $2 \mathrm{~cm}$ from the desired end point (target). The example of an arm movement is presented in Figure 10.

\section{DISCUSSION}

Indeed, it is very hard for such a simple network to learn the required movement for such a broad range of action. But, since the approximate torque-time profiles are built considering the equilibrium torque values, the controller was able to learn and execute the movement. However, this indicates the inherent problem in this strategy that the network output totally depends on the predefined torque-time schema. Thus, a schema that is supported by the experimental data should be used to achieve acceptable result.

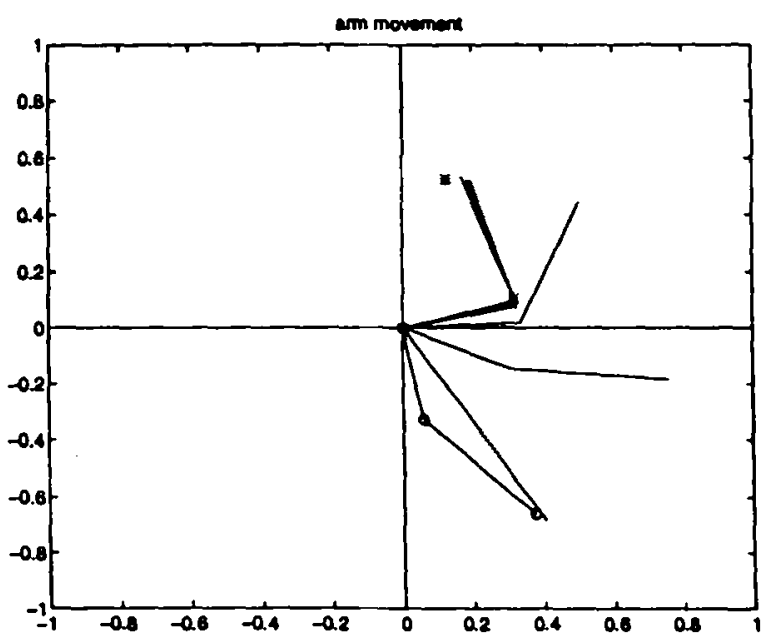

Figure 10. An example of a reaching movement performed by network. The stars represent the desired final position while the open circles represent the initial arm position.

The supervised learning of the static equilibrium torques could have easily been replaced by the analytical closed form solution, however, the following approach may be more physiological that the internal mapping between the required torque to maintain a given posture is learned (perhaps with the assistance of the many mechano-sensors that musculoskeletal system is endowed). The NN1 learns to control according to the reinforcement signal. The task, the range of the working space, the torque-time profiles and the reinforcement signal should all be consistent. Although all the necessary information should be provided in the reinforcement signal to inform the network regarding different aspects of the system performance, these multiple, complex and competing metrics may cause confusion in learning process. Thus, one should be careful in defining the reinforcement signal.

In this study, we only evaluated the final distance of the arm endpoint from the desired endpoint. Thus, no extra information about the trajectory that the arm should follow is given. The following first step allowed generation of feasible torque profiles meeting the overall gross objectives of reaching the targeted final position. However, future studies must incorporate the various constraints that are present on the neuromusculoskeletal system and attempt to further optimize the performance based on various physical or physiological criteria (i.e. minimum jerk, minimum energy or minimum fatigue) [6].

While this study does not identify the biological systems carrying out these learning processes, Shadmehr and his colleagues have provided evidence for generation of internal model of the task as formation of motor memory that associates a desired trajectory of the limb to the required muscle torques [7,9]. They 
have suggested that the brain may control movement by combination of motor primitives, where each primitive is an element of computation in the sensorimotor map that transforms desired limb trajectories into appropriate motor commands [9]. They postulated that the activities of Purkinje cells in cercbellum may encode primitives that underlie the learning dynamics based on the resemblances of the mathematical properties of the derived primitives and the tuning curves of Purkinje cells in the cerebcllum. They have indicated that within a short time window after completion of the practice of a motor task, the critical events for representation of the learned skill to the central nervous system takes place based on their investigation of neurophysiological and functional changes they observed in their experiments studying the consolidation of the motor memory $[7,9]$.

\section{REFERENCES}

1. Flash $\mathrm{T}$, Hogan $\mathrm{N}$ : The coordination of arm movements: an experimentally confirmed mathematical model. J Neuroscience, 1985,5(7),1688-1703.

2. Gullapalli V: A Stochastic reinforcement algorithm for learning real valued functions. Neural Networks, 1190,3,671-692.

3. Gullapalli V: Reinforcement learning and its appli- cation to control. COINS Technical Report 92-10, Jan 1992.

4. Hertz J, Krogh A, Palmer RG: Introduction to the Theory of Neural Computation, Addison Wesley, 1991.

5. Pandy $M G$, Berme N: A numerical method for simulating the dynamics of human walking. $J$ Biomechanics, 1988, 21(12), 1043-1051.

6. Parnianpour M, Wang JL, Shirazi-Adl A, Khayatian B, Lafferriere G: A computational method for simulation of trunk motion: Towards a theoretical based quantitative assessment of trunk performance. Biomedical Engineering, Applications, Basis and Communication. 1999, 11(1):27-38.

7. Shadmehr R, Thoroughman $K$ Learning and memory formation of arm movements. In: Biomechanics and Neural Control of Movement, JM Winters and PE Crago (eds), New York: SpringerVerlag, 2000, pp. 347-353.

8. Sutton RS, Barto AG, Williams RJ: Reinforcement learning is direct adaptive optimal control. IEEE Control Systems Magazine, 1992, 12(2),1922

9. Thoroughman KA, Shadmehr R: Learning of action through adaptive combination of motor primitives. Nature 2000,407:742-747.

10. Winter DA: The Biomechanics and Motor Control of Human Gait. University of Waterloo Press. 1987 